\title{
'Touchpoints' by nurses: impact on maternal representations, child development, quality of mother-infant interaction, and mothers' perception of the quality of relationships with nurses
}

\begin{abstract}
Aim: To investigate the effect of implementing the Touchpoints methodology by nurses in the following variables: quality of mother-infant interaction; infant development; maternal representations of child temperament and mothers' perception of the quality of relationship with nurses.
\end{abstract}

\begin{abstract}
Methods: Quasi-experimental longitudinal study, including 86 child-mother dyads distributed equally for: Group with Intervention (GI) (n=43), Group without Intervention (GWI) (n=43). These groups belonged to paired samples according to the following criteria: maternal age; socio-economic class; family structure; child health; parents' physical or psychological health; twins; family's nationality; risk during pregnancy; baby APGAR. Paired samples with the same routine visits allowed comparing the impact of Touchpoints intervention on the above mentioned variables. The monitoring of the two groups took place in a period of between 11 and 24 months of children's life (four moments of assessment), being held two Touchpoints sessions in the Gl at 12 and 18 months. Two Touchpoints interventions sessions were applied in the $\mathrm{Gl}$ as follows: the first time, at 12 months; the second time, at 24 months, child age.
\end{abstract}

The instruments used for data collection were: Schedule of Growing Skills II (SGS II); CARE-Index; Temperament Scale; Parent-Caregiver Relationship Scale - parents' version.

Results: Infant Locomotor development $(\mathrm{p}=.036)$ and maternal representations about the child and motherhood ( $Z=5.737 ; p=.019)$ improved in the Gl. No significant results were found for mother-infant interaction in this direct comparison. Nevertheless, findings indicate that maternal sensitivity and infant cooperative behaviour increased from 12 to 24 months in the Gl [t(41)=4.513; $p<.001$, whereas it decreased in the GWI (from 8.62 at 12 months to 8.40 at 24 months). The means of mothers' perceptions of Trust/Caring towards nurses in the $\mathrm{Gl}$ were higher than in $\mathrm{GWI}$ after six months of the Touchpoints intervention $[\mathrm{t}(84)=2.146$; $\mathrm{p}<.001 ;$ M_Gl=34.07, SD=3.71; M_GWl=31.79], Collaboration/Partnership [t(84)=2.817; $\left.\mathrm{p}<.001 ; \mathrm{M} \_\mathrm{Gl}=62.79, \mathrm{SD}=4.809 ; \mathrm{M} \_\mathrm{GW}=59.89, \mathrm{SD}=6.538\right]$ and Relational/Emotional $\left[t(84)=2.334 ; p<.001 ; M \_G l=60.53, S D=3.838 ; M \_G W I=58.30, S D=5.638\right]$.

Children at risk for developmental problems in the GI and GWI improved their development in Speech and Language $\left(Z=4.772 ; p<.05 ; M \_G l\right.$ at risk $=13.64 \mathrm{SD}=.30$ vs. M_GWl at risk=13.00, $S D=.29)$, Hearing and Language $\left(Z=4.341 ; p<.05 ; M \_G l\right.$ at risk=15.08, $\mathrm{SD}=.37$ vs M_GWl at risk=14.0, $S D=.36)$ and in Self-care Social $(Z=4.0 ; p<.05$ vs M_Gl at risk=11.96, $S D=.35 ;$ M_GWI at risk=11.46, $S D=.34)$ after the intervention with Touchpoints. Also, maternal sensitivity and infant cooperative behaviour increased after Touchpoints intervention in dyads of 'children at risk' for developmental problems when compared to the dyads of 'children at risk without' these problems.

Conclusion: The intervention using Touchpoints methodology by nurses had a positive effect on the following dimensions: infant development; maternal representations about the child and motherhood; maternal sensitivity and infant cooperative behaviour; perceptions of Trust/Caring towards nurses. This was particularly significant among children who were identified as having potential development risks. Further research is needed to better explain these findings. 